\title{
An integrated custom decision-support computer aided facility management informative system for healthcare facilities and analysis
}

\author{
Ernesto ladanza ${ }^{1}$ (D) $\cdot$ Alessio Luschi ${ }^{1}$
}

Received: 10 June 2019 / Accepted: 4 September 2019/Published online: 10 September 2019

(C) The Author(s) 2019

\begin{abstract}
This article presents a Computer Aided Facility Management informative system which can output Key Performance Indicators and quantitative parameters about the analysed healthcare facility. The designed system is a self-sufficient application able to manage and analyse digital plans of hospital buildings with no need of third-party plugins or licenses. The system maps hospital's inner organisation, destinations of use and environmental comforts giving quantitative, qualitative and graphical reports. The core database is linked to other existing hospital databases, so that the system can act as a central control cockpit. Outputs can be used by top-management and decisional staff as a decision-support tool in order to improve hospital's structure and organisation and to reduce the major workflow risks. Furthermore, many plug-ins and modules have been developed through the years which can be easily linked to the main application thanks to its REST architecture, and which contribute to a complete analysis and management of the healthcare facilities.
\end{abstract}

Keywords CAFM $\cdot$ Hospital $\cdot$ Healthcare $\cdot$ Decision-support $\cdot$ REST $\cdot$ Planning $\cdot$ Clinical engineering

\section{Introduction}

Modern healthcare facilities must deal with complex informative flows related to information with different sources and scopes. Furthermore, hospitals must be compliant with strict hygienic, qualitative and organisational standard requirements set by national and international institutions to accomplish clinical and medical duties. Clinical Engineering Services and Technical Departments must find solutions to fully satisfy all sorts of technological, structural and organisational needs for such a complex structure as a hospital.

Several technical tools have been developed for these needs to monitor the hospital just as well as any other sort of composite facility by measuring quantitative, architectonical, technological and people-related parameters.

Ernesto Iadanza

ernesto.iadanza@unifi.it

Alessio Luschi

alessio.luschi@unifi.it

1 Department of Information Engineering, University of Florence, Via S. Marta 3, 50139, Florence, FI, Italy
Many of these systems are based on applications of data management in Internet of Things (IoT) [1], where software and external machines interact to monitor the statusquo of complex networks such as logistics and supply chain management [2-4], disaster and ecological monitoring environment systems [5] and indeed, healthcare facilities monitoring systems [6-8]. Typically, data storages have to deal with a huge amount of data, and they can be identified as traditional Relational Database Management System (RDBMS) such as MS SQL Server or MySQL [9, 10], and NoSQL (Not only SQL) like MongoDB or CouchDB [11-13]. Regardless of the adopted data management architecture, data can be aggregated in different ways to answer to a wide range of queries. Numeric indexes have been proposed by the scientific community to monitor the performance of buildings so that quantitative analysis can be made for different healthcare facilities [14, 15]. These Key Performance Indicators (KPIs) can be automatically calculated starting from these data.

An evolution of simple DBMS is Geographic Information Systems (GIS) which are tools designed to capture, store, manipulate, analyse, manage and present all types of geographically referenced data. This is a technology that merges cartographies, statistical analysis and data. There are two specific types of data referring to a map, namely: 
- spatial data, which describe the place and the form of the geographical objects and their spatial relations with other objects;

- descriptive data, regarding the geographic objects (attributes).

Spatial data are rendered via graphical features: points, vectors and polygons. The attributes represent the qualitative or quantitative side of the rendered phenomenon on a digital map. Thus, for a geographical entity, the attribute table can associate different numerical and textual parameters. GISrelated software usually performs typical measurements like path-analysis and people finding but generally provides no quantitative comparable data [16, 17].

Computer Aided Facility Management (CAFM) systems are decision-support tools based on Integrated Healthcare Facility Management Models (IHFMM) which provide KPIs on those processes which can affect the performance of the healthcare structure. These tools can be very useful for decision makers for performance and risk evaluations, business management and development $[18,19]$.

Workplace Management Systems (WMS) are solutions designed to manage real estate facilities, allowing users to assess, analyse and reorganize the company assets so that their value can be preserved. They are also very useful for improving effectiveness and responding to multiple needs. They provide access to stored information regardless of the workplace: data and plans can be acquired via web-services, using a common browser over an Internet or intranet network [20, 21]. These systems usually drive a Computer-Aided Design (CAD) engine to store information about space-units, assets, plants, phones, data plugs, wirings, etc., giving visual outputs.

Building Information Modelling (BIM) approach is nowadays becoming very common, and sometimes mandatory, for designing hospitals as well as for managing these structures throughout their life span. Not just spatial data, but also structural functional data could be addressed using this approach [22-24].

However, implementing a complete BIM model for such a complex scenario like a healthcare structure requires more resources than the traditional approach. Moreover, it usually does not cover external assets or equipment [25].

The main informative unit, i.e. the maximum degree of detail, may be a homogeneous functional area (a set of rooms pooled together by destination of use) or a single room. The first approach offers a useful overall view but does not allow accurate information on single room's supplies. The second one is instead a more full-scale methodology.

This article presents a WMS integrated tool which outputs KPIs and quantitative parameters typical of CAFM systems that allow to assess the entire building or technological estate. Priorities to most urgent interventions can also be assigned.
Despite being an appealing and popular concept nowadays, it has been decided not to use BIM solution for acquiring information about the buildings and data. In fact, BIM systems are developed focusing mostly on construction work and, although they provide final users with appealing $3 \mathrm{D}$ views and nice presentation, they are not mature enough when it comes to health technology management.

With that been said, the architecture of the presented system relies on importing plain 2D maps, one for each floor and pavilion, like a typical CAFM system. This is because of the intention to offer a central management cockpit which deals not only with structural and constructional data, but also with technologies, assets and medical equipment [26-28].

However, the main difference between the proposed system and a standard CAFM is the independency from any CAD software. Indeed, the presented informative system is able to convert DXF (Drawing Exchange Format) files into HTML5compliant Scalar Vector Graphics (SVG) drawings. Then it directly exploits this format for implementing CAD and WMS functionalities, without any further needs to access the initial map. This implies that no external license or software is needed in order to have the system fully operating, allowing an easier maintenance and a potential saving of external resources and funds.

The developed system is a suite consisting of a main software module named SPOT and extra-tools which all refer to the same inner database linked to the Hospital Information System (HIS) (Fig. 1). It is made by a stand-alone main executable application which monitors the status-quo of the buildings in terms of beds, square meters, destination of use, functional areas and many other features for every room. The main module is only for technicians while aggregated and already

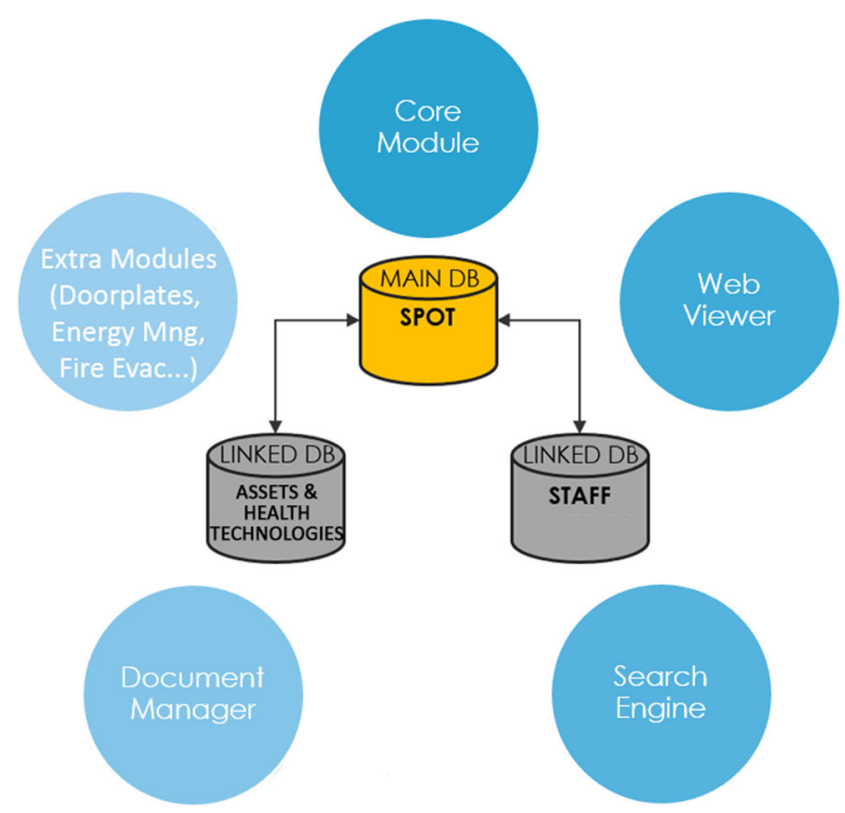

Fig. 1 Designed CAFM system relational schema 
evaluated data are shown to hospital's staff and managers by using a RESTful (REpresentational State Transfer) HTML5compliant rendering engine and layout available via webbrowser named SPOTWEB. It can be also described as a central dashboard: indeed, the main database is linked to other hospital datastores, allowing aggregation among heterogenous and initially unlinked information. Furthermore, SPOT FINDER is a web-based search-engine which allows users to perform free-text queries on the data stored in the database. The system is also provided with a real-time reporting engine. Moreover, DOCUMENT MANAGER is an additional module used to link external digital attachments to rooms or functional groups, regardless of formats and sources. All the modules have been designed and developed at the Department of Information Engineering of the University of Florence within a research program applied to the hospital campus of the Azienda Ospedaliero-Universitaria Senese (AOU Senese) in Siena, Italy.

\subsection{Organization of the azienda ospedaliero-universitaria senese}

The AOU Senese is a pavilions-hospital campus with 12 different buildings over more than 200,000 square meters, with about 800 beds and 8100 rooms. The hospital represents a peculiar case-study, because of its outer spatial dislocation. Pavilions are built in an area with a craggy discontinuous topography. This does not allow inner paths and alleys to be on the same constant level throughout the area. For example, it is not rare to have the first basement of a given building connected with the first floor of an adjoining pavilion through the same hallway.

Hospital's inner organisation is today structured in 27 Departments and 217 Operative Units. Departments are functional macrostructures associated with the clinical and nonclinical supplied offer. Functional substructures with a lower level of aggregation called Operative Units (OU) are defined for each department. They can be viewed as pools of physicians who share a specific set of spaces in a tier. Therefore, a single room could also be assigned to more than one OU (this is quite common in case of outpatient clinics and surgery rooms, often used in many cross-specialties).

\section{Materials and methods}

\subsection{Spot main module}

The idea behind this work is to bring the knowledge-sharing to its maximum, allowing staff to know as much as possible about their hospital. Despite being a CAFM system, the designed application does not directly drive CAD software (e.g. AutoCAD, ArchiCAD, etc.) but it uses inner libraries to convert DXF digital plans (which can be exported by most CAD software) into SVG so that they can be displayed and managed with nothing but common HTML5-compliant webbrowsers (Google Chrome, Mozilla Firefox, Microsoft Edge, Apple Safari, etc.). The only requirement for the conversion to succeed is to have a dedicated layer on the DXF map (whose name can be set inside the system) with closed polylines, each one of them outlining a single room. Coordinates are saved straight inside the central database, which all data are stored in. Once the conversion has taken place, the starting DXF is no longer needed. Besides, all the graphical information about walls, windows, doors and any other architectonical element is also converted to SVG, so that the final user is able to look at the floor map in its integrity.

Once the import has been completed, the user is able to input information about different listed data for every room (see Table 1).

Data can be collected through on-site surveys and interviews to personnel, then spaces are classified by their usage and by customer expectations in terms of environmental comfort. Survey information is then data-entered into the system according to Table 1 . The system automatically assigns colours to rooms, according to the associated Destination of Use, Department, Operative Unit or Cleaning Class. The colour code is fully customisable in the software settings section. This allows users to immediately identify the main information about a room by simply looking at the floor map.

Collected information does not only allow the evaluation of spatial indicators (such as available surface for bed, number of elevators for bedridden patients, etc.), but also lets the decision makers manage the overall yearly cost for each space (for instance also by knowing its specific Cleaning Class and the related cost per square-meter). Information about the actual availability of spaces can be also managed, knowing for example the time periods when the rooms were not operational (e.g. for building yards).

\section{Spot web}

One of the main criticalities of a CAFM system is the usability of the application because it is accessed by heterogeneous users with different background, training and aims (top-management, engineers, nurses, physicians, technicians, external companies, etc.). A stand-alone software to be installed on local workstations would be nowadays a very inefficient solution for such a scenario. A server-based application with a central engine reached via web-browser is a better solution by far.

The designed viewer has this exact scope, offering a RESTful Web Application Programming Interface (API) to access all the available information, thus being complementary to the core module. 
Table 1 Managed listed data

\begin{tabular}{|c|c|}
\hline Data Name & Data Description \\
\hline Room Code & $\begin{array}{l}\text { A unique alphanumerical code to identify the building, the floor and the number of a } \\
\text { given room according to the rule PREMISE_BUILDING_LEVEL_ROOM, where } \\
\text { PREMISE is a single letter which identifies the premise, BUILDING is a four-digit } \\
\text { unique code for the building, LEVEL is a two-digit number for the floor (optionally } \\
\text { with a minus sign in front for basements) and ROOM is a 4-digit formatted incremental } \\
\text { alphanumeric code. The juxtaposition of these } 4 \text { codes gives a unique "talking code" } \\
\text { which identifies every single room inside the hospital itself letting the user immediately } \\
\text { know the position. }\end{array}$ \\
\hline $\begin{array}{l}\text { Destination of Use } \\
\text { (DU) }\end{array}$ & $\begin{array}{l}\text { A set of } 36 \text { categories every room can be assigned to, in order to straightly identify the } \\
\text { carried-on activity. }\end{array}$ \\
\hline Class & $\begin{array}{l}\text { A subset for every Destination of Use, used to narrow down the information about the } \\
\text { activity (for example, the Operating Room DU groups heterogeneous areas, which } \\
\text { must be differently treated both from architectural and engineering perspective. Classes } \\
\text { help to discriminate among "Patient Filter Zone", "Operators Filter Zone", and so on). }\end{array}$ \\
\hline Department & The medical or technical department the room is assigned to. \\
\hline Operative Unit & The Operative Unit assigned to the room. \\
\hline Cleaning Class & $\begin{array}{l}\text { A set of } 6 \text { classes associated with the complexity of the cleaning duties (Very High Risk, } \\
\text { High Risk, Medium Risk, Low Risk 1, Low Risk 2, Unapplied). The higher the } \\
\text { complexity, the higher the cost of the cleanings. }\end{array}$ \\
\hline Number of beds & The number of beds inside the room (where applicable). \\
\hline Surface & $\begin{array}{l}\text { The surface of the room automatically evaluated by its coordinates via Gauss algorithm } \\
\text { (sqm). }\end{array}$ \\
\hline Height & The height of the room (m). \\
\hline Volume & The volume of the room evaluated by its surface and height $\left(\mathrm{m}^{3}\right)$. \\
\hline Room Status & The current status of the room according to its actual usage. \\
\hline Maintenance Index & $\begin{array}{l}\text { A numeric index which highlights the complexity of the maintenance for that given room. } \\
\text { It is linked to the Destination of Use, but can be overridden by users with } \\
\text { administrative privileges. }\end{array}$ \\
\hline Personnel & Information about the people who work in that space, identified by their personal ID. \\
\hline
\end{tabular}

Maps are displayed by using automatically generated SVG polygons drawn from the room's coordinates stored in the core database. SVG format (W3C HTML5 compliant) can be viewed by any web-browser with no need of third-party plugins.

The application allows users to visualise the last updated map of the requested floor of a building in real time by using AJAX and Javascript. Every time a click event is triggered, it invokes an asynchronous access to the web-server via webservices. Then the controllers handle the request querying the database and responding with the requested information. Standard formats like JSON and XML are used.

The engine also provides the basic functions of a CAD engine such as multi-selection, panning, zooming, text placement and scaling (Fig. 2).

Moreover, the user can access information stored in the main database or in other linked databases according to the privileges associated with its username and with the rooms selection. This includes general and organisational details (Fig. 3) or specific details about available or installed assets and health technologies (Fig. 4).

The links between the databases are made by using the unique room code as identity key, so that heterogeneous information can be aggregated with just simple one-to-many links. This approach makes SPOT a convenient tool for accessing all sort of information available through several data sources, with a single central graphical web user-interface.

One further important feature the viewer must provide, is the possibility to navigate through the floors of the hospital in a horizontal way. This is an almost mandatory requirement because of the misalignment among the floors of different pavilions discussed above. Indeed, the user must be able to easily notice if and which level of a different building has a horizontal link to the current displayed floor, and then possibly navigate it (Fig. 5).

Structural data (surfaces, heights and beds), organisational data (name and contacts of the personnel, room code, organisational heterogeneous subgroups such as Departments, Operative Units and Cleaning Classes), technical data (medical devices and assets) as well as plants and architectural data (air treatment units, frames, lights) are all available for the final user.

\subsection{Spot finder}

Due to the great amount of data stored inside the database the CAFM relies on, a web search-engine is required to perform free-text queries according to unpredictable user's needs. The 

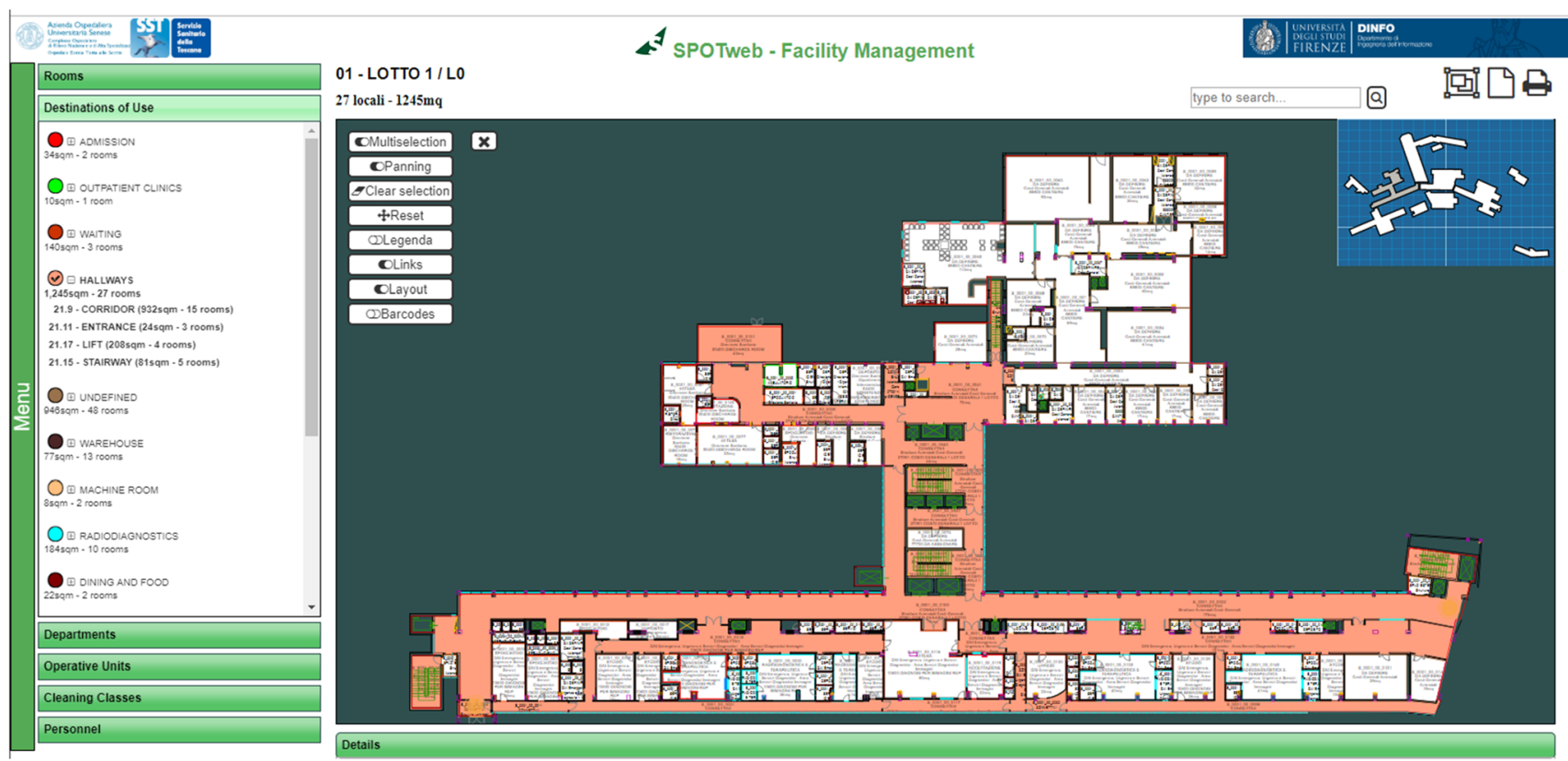

Fig. 2 Screenshot of the web-viewer. In the example all the hallways of the floor are selected (highlighted in colour)

designed engine is integrated within the web-viewer via dedicated web-service, but it also has its own GUI (Graphic User Interface) to perform more detailed searches, which can be even refined by using an advanced search tool to restrict the output (Fig. 6).

A search algorithm allows users to perform queries using the common Google syntax and ASCII characters (include, exclude, perfect search). Fuzzy Dictionary and Google APIs are also implemented to offer a "Did you mean" functionality.
The engine also provides a dynamic reporting function, which allows users to output custom reports based on the search results with different levels of aggregation.

\subsection{Spot document manager}

A problem which the executive board must often deal with when it comes to hospital management, is how to group together heterogeneous information not only in terms of subjects, but also in terms of formats and sources. For instance,
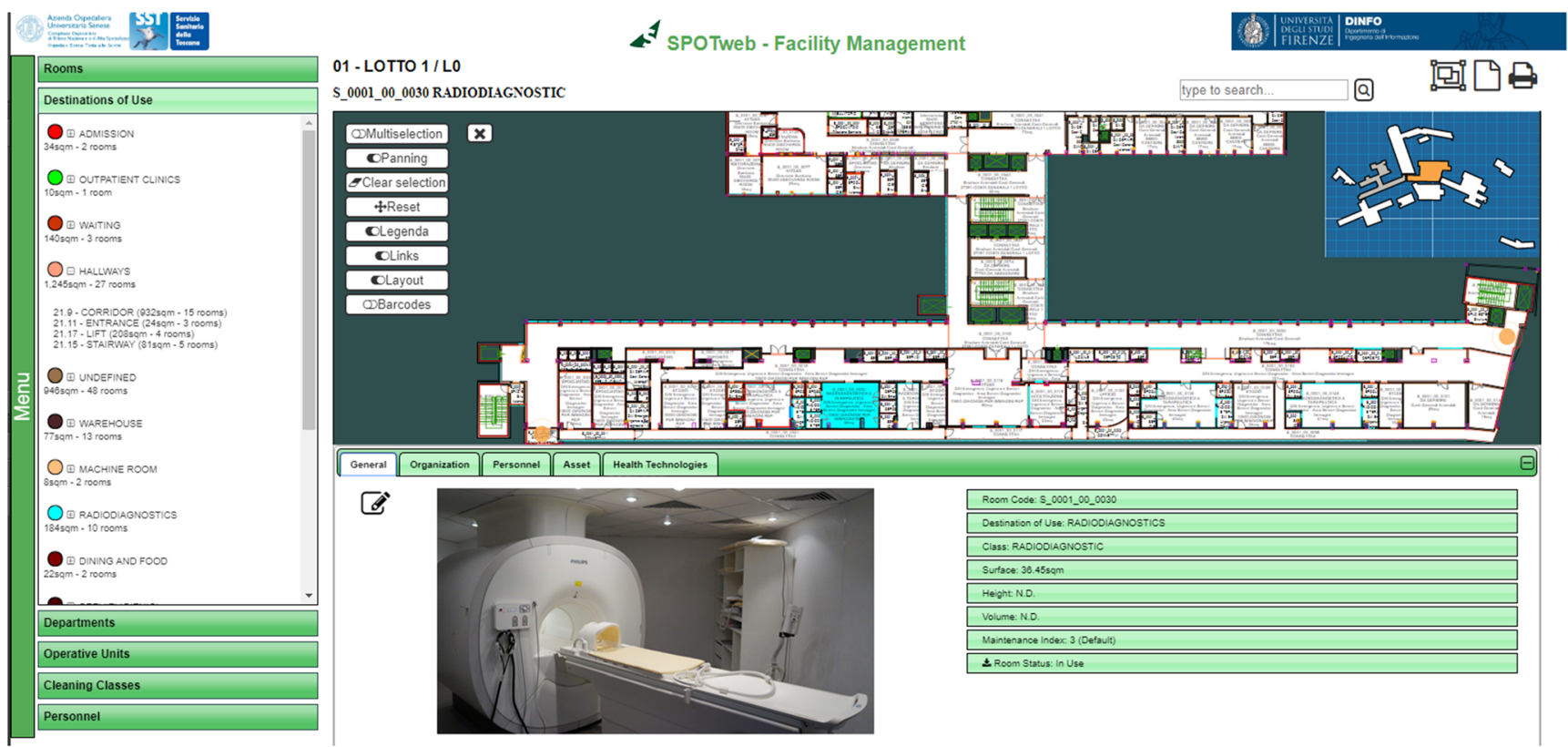

Fig. 3 Screenshot of the web-viewer. General details displayed in the bottom window. The accordion control on the left shows the functional group of the available Destinations of Use for the selected floor 


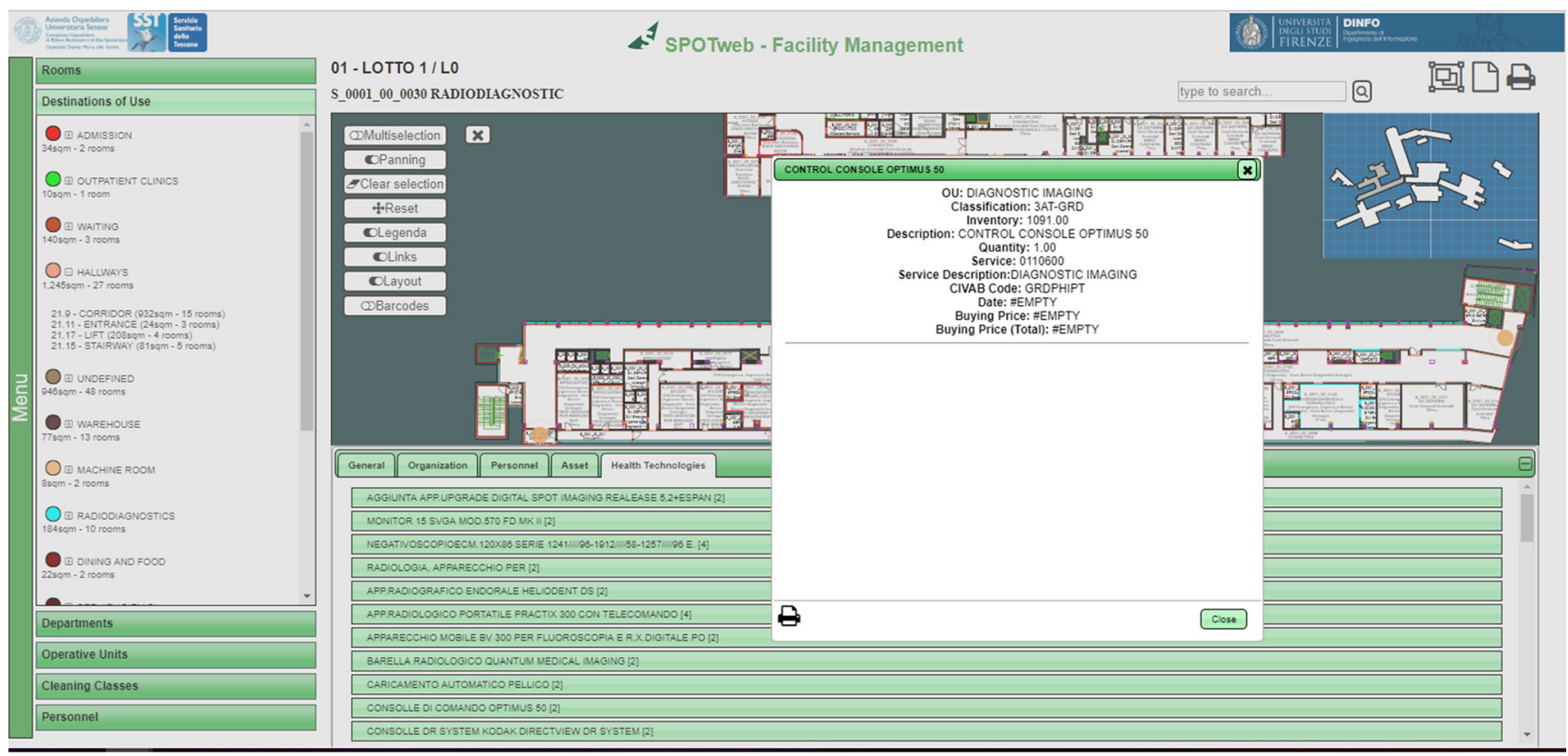

Fig. 4 Screenshot of the web-viewer. Detailed information about a Radiodiagnostic Control Console for the selected room (Room Code is S_0001_00_ 0030)

some text documents (e.g. meeting minutes or on-site inspection reports) may come in .pdf format and refer to a given organisational structure; reports may be .csv files with tables and plots about the ongoing cost of maintenance of a CT located in a particular ward; as-built digital plans of a floor may come in .dwg or any other CAD format. These are just few examples of how mixed and thus harder to admin than usual - the information management could be.
DOCUMENT MANAGER is on optional module specifically designed to mitigate this problem, helping offices and secretaries in digital document organisation, saving time for retrieving them by top-management or whoever needs them.

Files with any kind of extension and format can be uploaded to the system by using the above described webviewer interface, and can be linked with a single room or a functional subgroup like Departments, Operative Units or Cleaning Classes for a given floor and building.
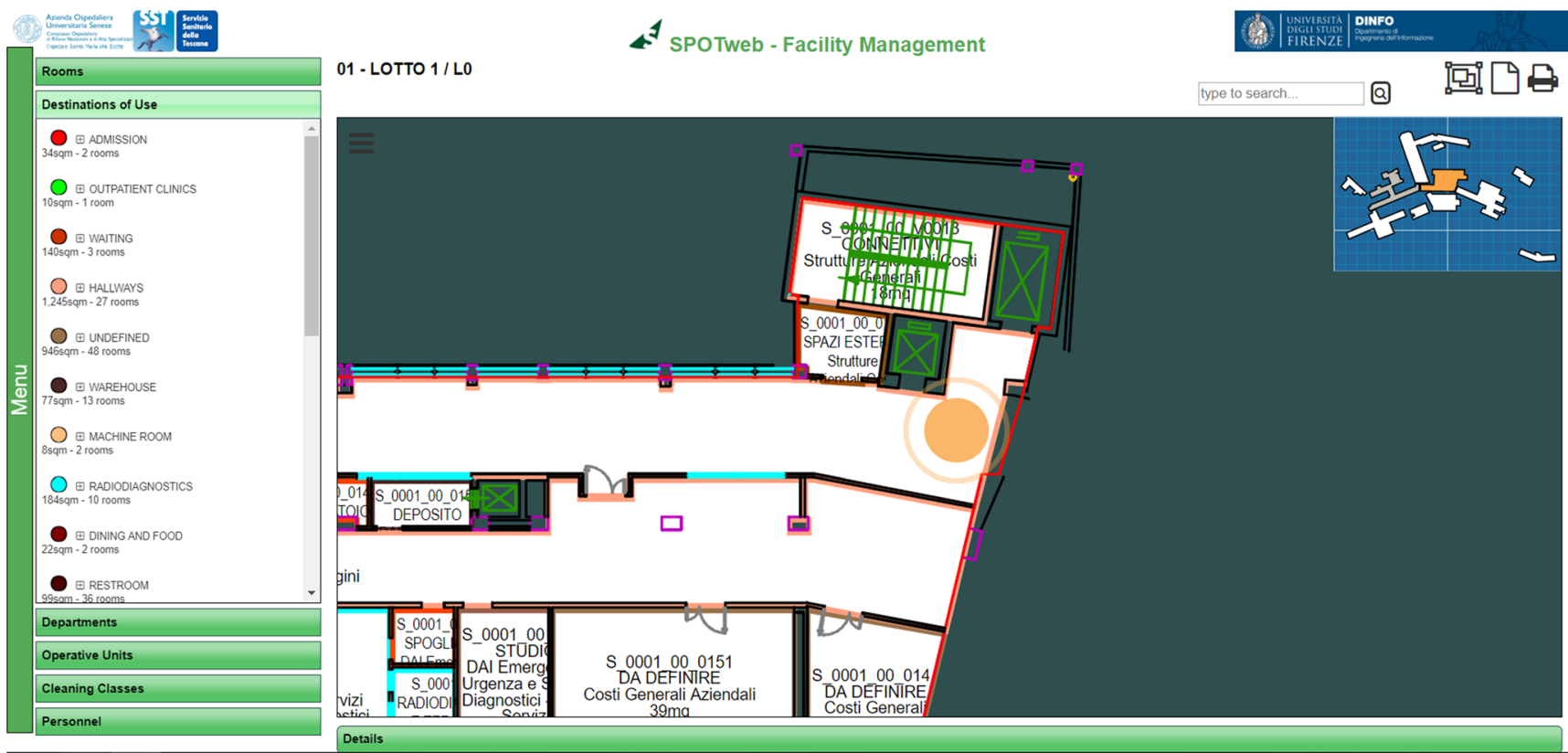

Fig. 5 The red arrow shows an existing link (a pulsing orange circle) at the end of the hallway to a different level in another adjoining pavilion (also highlighted in orange in upper-right navigator) 


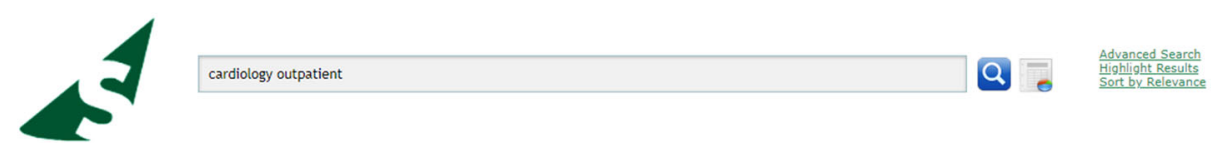

Did you mean:

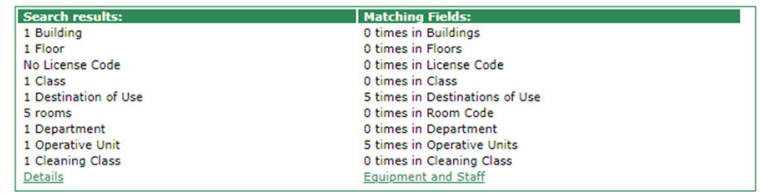

\begin{tabular}{|c|c|c|c|c|c|c|c|c|c|c|c|c|c|c|}
\hline Premise & Building & Floor & Room Code & Destination of Use & Class & Personnel & Dept & License & ou & Cleaning Class & Beds & Surface & Height & \\
\hline LE SCOTTE & LOTTO2 2 & $\lfloor 2$ & 50002020055 & OUTPATIENT CLINIC & ECHOCARDIOGRAPHY & & Cardiology Department & & 42100 - CARDIOLOGY WARD & MR - Medium Risk & & 23.77sam & & 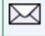 \\
\hline LE SCOTTE & LOTTO 2 & $\underline{12}$ & $\$ 0002020056$ & OUTPATIENT CLINIC & ECHOCARDIOGRAPHY & & Cardiology Department & & 42100 - CARDIOLOGY WARD & MR - Medium Risk & & $13.19 \mathrm{sqm}$ & & $\square$ \\
\hline LE SCOTTE & L्OT102 & $\underline{12}$ & 50002020058 & OUTPATIENT CLINIC & ECHOCARDIOGRAPHY & & Cardiology Department & & 42100 - CARDIOLOGY WARD & MR - Medium Risk & & $17.62 \mathrm{sam}$ & & 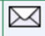 \\
\hline LE SCOTTE & L्वाт्2 & $\lfloor 2$ & $\$ 0002020056$ & OUTPATIENT CLINIC & ECHOCARDIOGRAPHY & & Cardiology Department & & 42100 - CARDIOLOGY WARD & MR - Medium Risk & & $20.13 \mathrm{sqm}$ & & 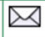 \\
\hline LE SCOTTE & LOTIO 2 & $\underline{12}$ & $5 \quad 0002020082$ & OUTPATIENT CLINIC & ECHOCARDIOGRAPHY & & Cardiology Department & & 42100 - CARDIOLOGY WARD & MR - Medium Risk & & $19.92 \mathrm{sqm}$ & & -4 \\
\hline
\end{tabular}

Fig. 6 Screenshot of the search engine. In the example the results for keywords "cardiology outpatient" are displayed

Afterwards, users log into the application by using a different dedicated GUI in order to visualise a folder structure for the uploaded attachments grouped by pavilion, level, organisational area and room, while numerical badges next to the folders name easily identify the number of uploaded documents (Fig. 7). In the upper side of the interface a search-bar is available to perform textual searches through the tree of attachments. When a file is selected, a logged user can download it or - if provided with high privileges - also delete it.

Rooms with attached documents, or being part of a functional subgroup with attached documents, are also highlighted in the web-viewer by a paperclip icon next to the name.

\subsection{Doorplate module}

The last available module of the suite is the doorplate production module. By using the main application, the user assigns personnel to each room in the facility (as shown in Table 1). The central database is linked to the hospital staff one where people are associated with an Operative Unit. Staff members are firstly filtered by choosing the Operative Unit for the analysed room, and then linked to the room itself. Once the link is performed, the system can automatically generate doorplates for the selected rooms, with predesigned layouts according to the Destination of Use and to the building (Fig. 8).

\subsection{Additional modules}

Besides the described functionalities, the REST approach allows additional modules to be implemented and linked very easily [29]. In this regard various additional features have already been designed and tested:

- ENERGY MANAGEMENT system is able to interface with an existing sensor system via asynchronous calls and web-services, in order to manage the compliance of the actual environmental parameters for a single room (temperature, humidity and air ventilation) to the legislative mandatory standards, according to its Destination of Use and Environmental Class [30, 31].

- HOSPITAL SCHEMA BUILDER is a transfer management tool which helps the top-management assessing the impact of complex transfers of whole Operative

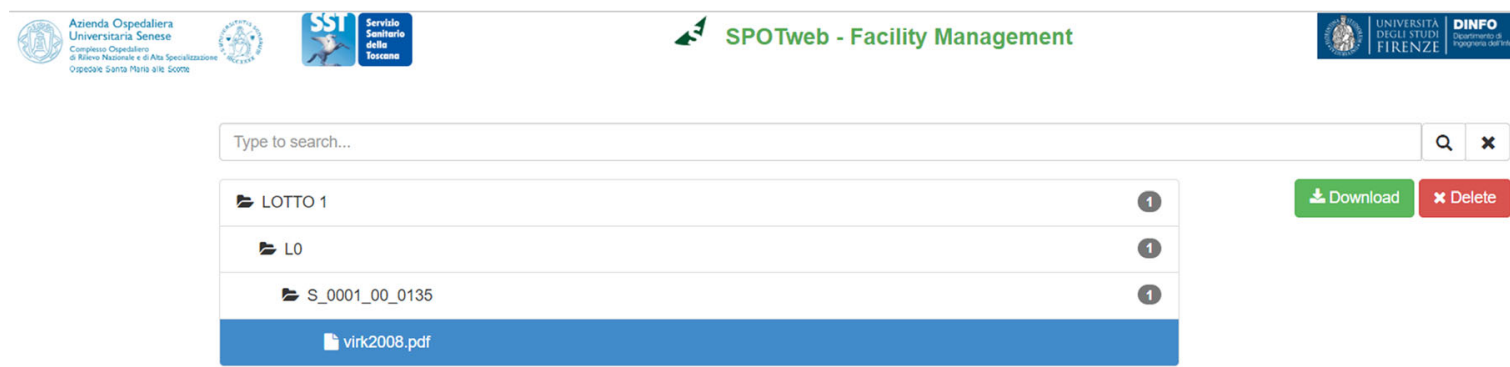

Fig. 7 Document Manager GUI with administrative login privileges 


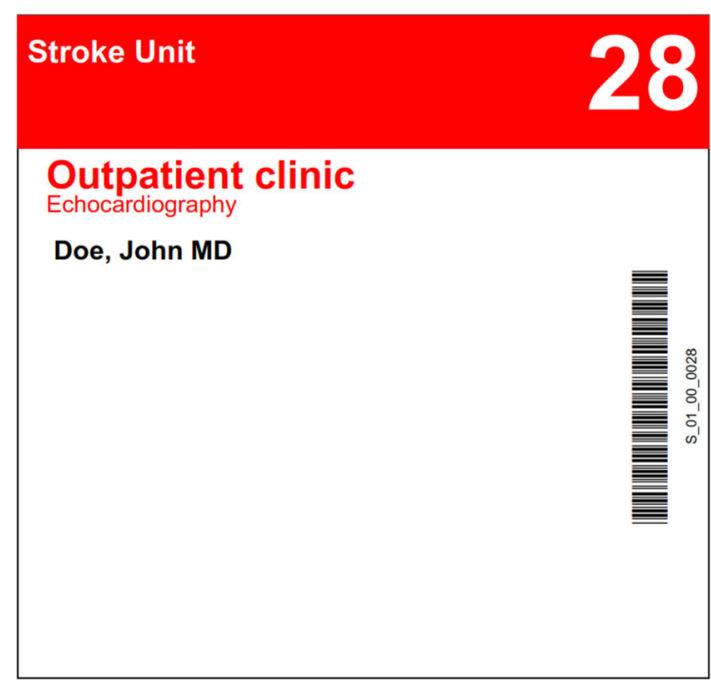

Fig. 8 Available doorplate layouts

Units or Departments from one location to a new on. The hospital is visualized in terms of functional organisation rather than spatial, where each cell is a single OU, grouped by level and building in a matrix shaped layout [32].

- BED MANAGEMENT system is a designed module which provides the real time picture of the beds availability for a given ward, analysing the interactions between patients, admission status, housekeeping staff and physicians, lowering the length of stay and the cost of care [33].

- FIRE EVACUATION system evaluates the safest evacuation path from a ward in case of fire emergency, analysing the architectonical floor layout, the possible bottlenecks and patients' speed according to their illness and admission, in relation to the position of the fire trigger and its time progression [34].

- LICENSING system automatically verifies the compliance of a ward with legislative mandatory structural and technological requirements, by analysing data stored inside both the main and the linked databases together with the architectonical layout of the SVG maps [35].

\subsection{Languages, frameworks and DBMS adopted}

SPOT main relational database is a Microsoft SQL Server 2008 R2 instance installed on one of the many available hospital data-servers. The core module has been developed in Visual Basic as a Windows Forms Application, within Microsoft. NET Framework 4.5.2, both for 32bit and 64bit architectures.

SPOTWEB, SPOT FINDER and DOCUMENT MANAGER are developed in Visual Basic as a MS. NET Framework 4.5.2 Model-View-Controller (MVC) application installed on a

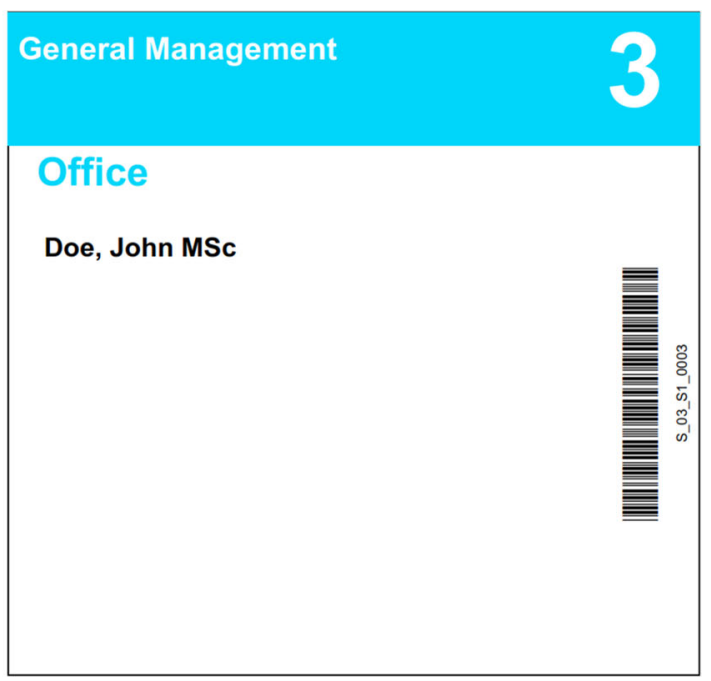

web-server running on the hospital network. The interaction with the main database is made using Entity Framework (EF) as Object Relational Mapper. Standard Javascript, jQuery library and Bootstrap framework are used for client-side programming, for displaying functions and for AJAX calls to the Web APIs. D3.js library has been used to implement CAD functionalities like panning and zooming on the SVG drawings. Finally, Poppler and Cairo libraries based PDF2SVG were used to allow conversion of the architectonical layout for a single level from PDF to SVG format [36].

\section{Results}

The informative suite is applied to the above mentioned AOU Senese hospital campus in Siena. It is used on a daily basis by several hospital offices, especially by the General Management, the Clinical Engineering Service and the Technical Department and it is constantly updated by designated personnel. The SPOT intranet weblogs reveal about 600 hits by 85 unique visitors per day.

The system allows queries on rooms outputting both numerical and graphical reports (see Fig. 9), therefore becoming a support tool for the healthcare planning.

A list of few examples, grouped by users, follows:

- Directors and Nurse Coordinators use SPOTWEB in conjunction with SPOT FINDER to know the spatial distribution of the units/departments under their control, the availability of medical equipment and assets. They query the system also to retrieve the inventory number of a device for scheduled or corrective maintenance purposes.

- Health and Safety Service queries SPOT (in particular the FIRE EVACUATION additional module) to know the escaping pathways along the buildings, where the fire- 


\section{Destination of use}

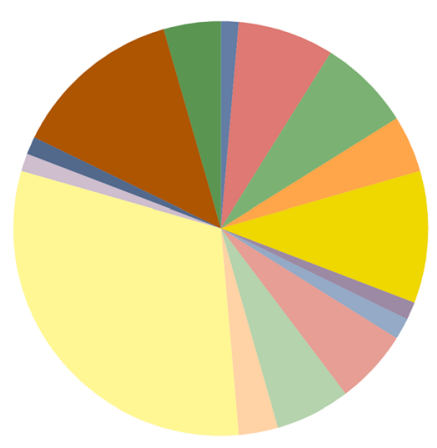

\begin{tabular}{lrr}
\hline ADMISSION & 1 & $1,5 \%$ \\
OUTPATIENTS & 5 & $7,4 \%$ \\
HALLWAYS & 5 & $7,4 \%$ \\
WARDS & 3 & $4,4 \%$ \\
WAREHOUSES & 7 & $10,3 \%$ \\
DIDACTICS & 1 & $1,5 \%$ \\
WORKSPACES & 1 & $1,5 \%$ \\
MACHINE ROOMS & 4 & $5,9 \%$ \\
RADIODIAGNOSTICS & 4 & $5,9 \%$ \\
DINING AND FOOD & 2 & $2,9 \%$ \\
RESTROOMS & 21 & $30,9 \%$ \\
LOCKER ROOMS & 1 & $1,5 \%$ \\
STERILIZATIONS & 1 & $1,5 \%$ \\
STUDIES & 9 & $13,2 \%$ \\
OFFICES & 3 & $4,4 \%$ \\
Total: & 68 & $100,0 \%$ \\
\end{tabular}

\begin{tabular}{|c|c|c|c|c|c|c|c|c|c|c|c|c|}
\hline Presidio & Edificio & Piano & Cod. Amb & Dest. d'uso & Classe & DIP & Accredit. & UU. 00. & PPLL & $M Q$ & Alt. & Cl. Pulizia \\
\hline LE SCOTTE & LOTTO 2 & L2 & $\begin{array}{c}\text { S_0002_02 } \\
-0055\end{array}$ & AMBULATORIO & $\begin{array}{l}\text { ECOCARDIOG } \\
\text { RAFIA }\end{array}$ & $\begin{array}{l}\text { DAI } \\
\begin{array}{c}\text { Cardio-Toraco-Vas } \\
\text { colare }\end{array}\end{array}$ & & $\begin{array}{l}\text { CARDIOLOGIA } \\
\text { UNIV.-REPARTO }\end{array}$ & & 23,77 & & $\begin{array}{l}\text { MR - Medio } \\
\text { Rischio }\end{array}$ \\
\hline LE SCOTTE & LOTTO 2 & L2 & $\begin{array}{c}\text { S_0002_02 } \\
-0056\end{array}$ & AMBULATORIO & $\begin{array}{l}\text { ECOCARDIOG } \\
\text { RAFIA }\end{array}$ & $\begin{array}{l}\text { DAI } \\
\begin{array}{c}\text { Cardio-Toraco-Vas } \\
\text { colare }\end{array}\end{array}$ & & $\begin{array}{l}\text { CARDIOLOGIA } \\
\text { UNIV.-REPARTO }\end{array}$ & & 13,19 & & $\begin{array}{l}\text { MR - Medio } \\
\text { Rischio }\end{array}$ \\
\hline LE SCOTTE & LOTTO 2 & L2 & $\begin{array}{c}\text { S_0002_02 } \\
-0057\end{array}$ & ACCETTAZIONE & $\begin{array}{c}\text { ACCETTAZION } \\
E\end{array}$ & $\begin{array}{l}\text { DAI } \\
\text { Cardio-Toraco-Vas } \\
\text { colare }\end{array}$ & & $\begin{array}{l}\text { CARDIOLOGIA } \\
\text { UNIV.-REPARTO }\end{array}$ & & 48,67 & & $\begin{array}{l}\text { MR - Medio } \\
\text { Rischio }\end{array}$ \\
\hline
\end{tabular}

Fig. 9 Numeric report of search results

escapes and fire-stairs are, and to test the escaping times for different wards and departments according to several fire simulations. Moreover, the system gives a precise detail on which places are more sensible, thus asking for more attention (rooms with combustive or oxidizing agents like wards or Intensive Care Units, super-magnet rooms of Magnetic Resonance Imaging departments, etc.) [37].

- $\quad$ Energy manager often uses the ENERGY MANAGEMENT system to know the relations between a conditioning device and its referenced air treatment unit, or to evaluate the compliance between theoretical and real values for a given room in order to plan interventions and maintenance.

- Technical staff uses SPOT almost every day to retrieve parameters used for managing purposes and to calculate performance indicators for quality of service.

- Everybody inside the hospital can query SPOT FINDER to get to know room codes and Operative Units of any medical or non-medical activities. This tool provides information about the personnel too, so that it becomes a useful tool for people-finding as well.

The designed management system is used for many purposes and in many different scenarios like transfer management, accreditation requirements assessment, health technologies and asset management and general designing and remodelling.

The system is also used in hospital facility management and governance activities like destinations of use verification and cost-space analysis of Operative Units. It offers a complete database with multifunctional data which allows many typologies of aggregation and enquiries by different categories of users, with the possibility to make more complex studies like cost-benefit analyses or comparative analyses.

\section{Conclusion}

The main aim of this work is to empower the sharing of knowledge among different typologies of user, designing a Workplace Management Informative System with decision support features and the typical functionalities of a CAFM tool, but without having external CAD software involved. Technological and procedural data are resident inside the system so that they can be accessed by anyone provided with the right privileges. This comes very useful because it promotes a continuous updating process, with changing claims coming from nurses, physicians, technicians, engineers or managers. This approach could be ideally complemented by vertical solutions for the inspection of medical devices such as [38, 39].

New technologies, such as machine learning techniques, are now under study for designing the future lines of research. Automatic classification of rooms could possibly be achieved by exploiting computer vision algorithms. This would significantly reduce the initial setup time for a new hospital, as well as increase the frequency of periodical massive update campaigns. 
Moreover, as soon as the new buildings will be directly modelled with BIM, intriguing new functionalities might be added such as virtual/augmented reality (VR/AR) applications for training personnel and for helping patients in being more aware of specific healthcare therapeutic paths.

Funding Information Research funded by the Azienda Ospedaliero Universitaria Senese, Siena, Italy.

\section{Compliance with ethical standards}

Conflict of Interest The authors declare that they have no conflict of interest.

Ethical approval This article does not contain any studies with human participants or animals performed by any of the authors.

Open Access This article is distributed under the terms of the Creative Commons Attribution 4.0 International License (http:// creativecommons.org/licenses/by/4.0/), which permits unrestricted use, distribution, and reproduction in any medium, provided you give appropriate credit to the original author(s) and the source, provide a link to the Creative Commons license, and indicate if changes were made.

\section{References}

1. Vongsingthong S, Smanchat S. A Review of Data Management in Internet of Things. Asia Pac J Sci Technol. 2015;20(2):215-40.

2. Morabito LAAIG. The Internet of Things: A survey. Comput Netw. 2010:2787-805.

3. Guo B, Poling AD, Poppe MJ. GIS/GPS in Transportation, Real World Experiences. http://proceedings.esri.com/library/userconf/ proc95/to250/p249.html. Accessed 10 Sept 2019.

4. Bakht H. mobile ad hoc networks Various problems and some solutions. School of Computingand Mathematical Sciences, Liverpool John Mores University. http://www.oocities.org/ humayunbakht/PMANET.pdf. Accessed 10 Sept 2019.

5. Xiao Y, Li W, Chen X-J, Liu B-Y, Wang L, Fang D-Y. An Immune Theory Based Health Monitoring and Risk Evaluation of Earthen Sites with Internet of Things. IEEE 2013(2013 IEEE International Conference on Green Computing and Communications and IEEE Internet of Things and IEEE Cyber):378-382.

6. Noueihed J, Diemer R, Chakraborty S, Biala S. Comparing Bluetooth HDP and SPP for Mobile Health Devices. IEEE 2010;2010 International Conference on Body Sensor Networks.

7. Bin S, Yuan L, Xiaoyi W. Research on Data Mining Models for the Internet of Things. IEEE 2010.

8. Shahamabadi MS, Ali BBM, Varahram P, Jara AJ. A Network Mobility Solution Based on 6LoWPAN Hospital Wireless Sensor Network (NEMO-HWSN). IEEE 2013(2013 Seventh International Conference on Innovative Mobile and Internet Services in Ubiquitous Computing):433-438.

9. Iadanza E. An Unconventional Approach to Healthcare (Geographic) Information Systems using a Custom VB Interface to AutoCAD. Proceedings of the 1st International Workshop on Mobilizing Health Information to Support Healthcare-related Knowledge Work. Available at: 10.5220/0001812800130019 2009.
10. Luschi A, Miniati R, Iadanza E. A web based integrated healthcare facility management system. (2015) IFMBE Proceedings, 45, pp. 633-636, 2015.

11. Phan TAM. Cloud Databases for Internet-of-Things Data. Aalto University; 2013:viii + 70 .

12. Li T, Liu Y, Tian Y, Shen S, Mao W. A Storage Solution for Massive IoT Data Based on NoSQL. 2012 IEEE International Conference on Green Computing and Communications,Conference on Internet of Things, and Conference on Cyber, Physical and Social Computing 2012.

13. Francesco MD, Li N, Raj M, Das SK. A Storage Infrastructure for Heterogeneous and Multimedia Data in the Internet of Things. 2012 IEEE International Conference on Green Computing and Communications, Conference on Internet of Things, and Conference on Cyber, Physical and Social Computing 2012:26-33.

14. Rodriguez E, Miguel A, Sanchez MC, Tolkmitt F, Pozo E. A new proposal of quality indicators for clinical engineering. Proceedings of the 25th Annual International Conference of the IEEE Engineering in Medicine and Biology Society, vol. 4, pp. 3598$3601,2003$.

15. Iadanza E, Dori F, Biffi Gentili G, et al. A hospital structural and technological performance indicators set. (2007) IFMBE Proceedings, 16, pp. 752-755, 2007.

16. Naves Givisiez GH. Hospital Demand: Using GIS and spatial analysis for estimation. Anais da XXIV IUSSP General Conference, 1, pp. 1-33, 2001.

17. Muresan F, Tirt DP, Haindu I. Specific features of GIS database for hospital management. An example for Bihor county. Geogr Tech. 2006;1(1):133-8.

18. Lavy S, Shohet IM. Computer-aided healthcare facility management. J Comput Civ Eng. 2007;21(5):363-72.

19. Luschi A, Monti M, Iadanza E. Assisted reproductive technology center design with quality function deployment approach. (2015). IFMBE Proc. 2015;51:1587-90.

20. InfoCAD.FM at http://www.infocad.fm. Accessed 10 Sept 2019.

21. Meirovich C, Mann P. Codebook for Planning, Procurement, Testing and Commissioning. Proceedings of $3^{\text {rd }}$ Global Forum on Medical Devices, 2017.

22. Ho SP, Tserng HP, Jan SH. Enhancing knowledge sharing management using BIM technology in construction. Scientific World Journal, 2013, Article ID 170498, 2013.

23. Linehan M, Andress B. Medical equipment and BIM. Advancing the planning process with building information modeling. Health Facil Manag. 2013;26(11):21-4.

24. Golden Gate Safety Network at http://comopview.org/ggsn. Accessed 10 Sept 2019.

25. Meirovich C, Nagy GG. Equipment installation: testing/commissioning. IFHE Digest. 2019;2019:49-51.

26. Badnjevic A, Gurbeta L, Jimenez ER, Iadanza E. Testing of mechanical ventilators and infant incubators in healthcare institutions. (2015). Technol Health Care. 2015;25(2):237-50.

27. Luschi A, Marzi L, Miniati R. Iadanza E. A custom decisionsupport information system for structural and technological analysis in healthcare. (2014). IFMBE Proc. 2014;41:1350-3.

28. Iadanza E, Chini M, Marini F. Electromagnetic compatibility: RFID and medical equipment in hospitals. IFMBE Proc. 2013;39:732735,2013

29. Luschi A, Belardinelli A, Marzi L, Frosini F, Miniati R, Iadanza E. Careggi Smart hospital: A mobile app for patients, citizens and healthcare staff. (2014) IEEE-EMBS International Conference on Biomedical and Health Informatics (BHI), pp. 125-128, 2014.

30. Iadanza E, Turillazzi B, Terzaghi F, et al. The Streamer European Project. Case Study: Careggi Hospital in Florence. (2015) IFMBE Proceedings. 2015;45:649-52. 
31. Luschi A, Di Franco R, Turillazzi B, Iadanza E. System for Monitoring Environmental Parameters in a Hospital Facility. (2020) IFMBE Proceedings. 2020;73:413-6.

32. Iadanza E, Luschi A, Gusinu R. Terzaghi F. Designing a Healthcare Computer Aided Facility Management System: a New Approach. (2020) IFMBE Proceedings. 2020;73:407-11.

33. Iadanza E, Luschi A. Ancora A. Bed management in hospital systems. (2019) IFMBE Proceedings. 2019;68(3):313-6.

34. Iadanza E, Luschi A, Merli T. Terzaghi F. Navigation algorithm for the evacuation of hospitalized patients. (2019) IFMBE Proceedings. 2019;68(3):317-20.

35. Iadanza E, Ottaviani L, Guidi G, Luschi A. Terzaghi F. License: Web application for monitoring and controlling hospitals' status with respect to legislative standards. (2014) IFMBE Proceedings. 2014;41:1887-90.
36. Barton D, pdf2svg at http://www.cityinthesky.co.uk/opensource/ pdf2svg. Accessed 10 Sept 2019.

37. Bernardini G, Paganelli F, Manetti M, Fantechi A, Iadanza E. Syrma: A tool for a system approach to risk management in mission critical systems, (2013) International Journal of Business Information Systems 13 (1), pp. 21-44, 2013.

38. Gurbeta L, Badnjević A. Inspection process of medical devices in healthcare institutions: software solution. (2017) Health Technol. 2017;7(1):109-17.

39. Gurbeta L., Badnjević A., Kurta E. eVerlab: Software Tool for Medical Device Safety and Performance Inspection Management. (2020) IFMBE Proceedings, vol 73, pp. 429-435, 2019

Publisher's note Springer Nature remains neutral with regard to jurisdictional claims in published maps and institutional affiliations. 